\title{
Estudio de la gestoforma del lanzamiento a canasta en la liga E.B.A. Study of shot technique in basketball in E.B.A. league
}

\author{
Javier García, Sergio José Ibáñez, Sebastian Feu, María Cañadas, Isabel Parejo \\ Universidad de Extremadura(España)
}

\begin{abstract}
Resumen: El objetivo fue analizar la relación existente entre la gestoforma de los lanzamientos en liga E.B.A. (liga española de baloncesto amateur) y una serie de variables recogidas durante la ejecución del mismo, i) periodo ii) cuarto, ii) eficacia, iv) presión defensiva, v) zona del campo, vi) rol del jugador que lanza, y vii) la acción previa que realiza. Se registraron un total de 8869 lanzamientos $(167,40 \pm 21,45)$. Se encontraron relaciones estadísticamente significativas entre gestoforma y: i) eficacia del lanzamiento $\left(\mathrm{c}_{(33, \mathrm{~N}=6598)}^{2}=365.287, \mathrm{p}<.01 ; C=.229, \mathrm{p}<.01\right)$, ii) presión defensiva $\left(\mathrm{c}^{2}{ }_{(77, \mathrm{~N}=6598)}=365.287, \mathrm{p}<.01 ; C=.229, \mathrm{p}<.01\right)$, iii) gestoforma y zona del campo $\left(\mathrm{c}_{(165, \mathrm{~N}=6598)}^{2}=2060.092, \mathrm{p}<.01 ; C=.488, \mathrm{p}<.01\right)$, iv) valor del lanzamiento $\left(\mathrm{c}^{2}{ }_{(11, \mathrm{~N}=6598)}=1148.784 \mathrm{p}<.01, C=.385, \mathrm{p}<.01\right)$, v) rol del jugador que lanza $\left(\mathrm{c}^{2}{ }_{(22) \mathrm{N}=5344}=226.979 \mathrm{p}<.01 ; C=.201, \mathrm{p}<.01\right), \mathrm{y}$ vi) acción previa $\left(\mathrm{c}^{2}{ }_{(55, \mathrm{~N}=4331)}=1307.575 \mathrm{p}<.01 ; C=.482, \mathrm{p}<.01\right)$. Estos resultados contribuyen a mejorar el diseño de las sesiones de entrenamiento, ajustándolas a la situación real de juego.
\end{abstract}

Palabras clave: Baloncesto, Lanzamiento, Gestoforma, Análisis Notacional.

Abstract: The purpose of this study was to analyze shot technique in basketball in E.B.A. league (Spanish Basketball Amateur league), examining the relationship that exists between shot technique and a series of variables registered during this action, i) period, ii) quarter, iii) efficacy, iv) defensive pressure, v) zone of court, vi) position of player that took the shot, and vii) the previous action. A total of 8869 shots were registered $(167,40 \pm 21,45)$. A significant relationship existed only between shot technique and: i) efficacy of the throw $\left(\mathrm{c}^{2}{ }_{(33, \mathrm{~N}=6598)}=365.287, \mathrm{p}<.01 ; C=.229, \mathrm{p}<.01\right)$, ii) defensive pressure $\left(\mathrm{c}^{2}{ }_{(77, \mathrm{~N}=6598)}=365.287, \mathrm{p}<.01 ; C=.229, \mathrm{p}<.01\right)$, iii) zone of court $\left(\mathrm{c}^{2}{ }_{(165, \mathrm{~N}=6598)}=2060.092, \mathrm{p}<.01 ; C=.488, \mathrm{p}<.01\right)$, iv) value of the throw $\left(\mathrm{c}^{2}{ }_{(11, \mathrm{~N}=6598)}=1148.784 \mathrm{p}<.01, C=.385, \mathrm{p}<.01\right)$, v) position of player that took the shot $\left(\mathrm{c}^{2}{ }_{(22, \mathrm{~N}=5394)}=226.979 \mathrm{p}<.01 ; C=.201, \mathrm{p}<\right.$ $.01)$, and vi) the previous action $\left(\mathrm{c}^{2}{ }_{(55, \mathrm{~N}=4331)}=1307.575 \mathrm{p}<.01 ; C=.482, \mathrm{p}<.01\right)$. These results contribute to improve the design of the sessions of training, adjusting them to the real situation of game.

Key words: Basketball, Shot, technique, Notational analysis.

\section{Introducción}

Los continuos esfuerzos por conseguir la mejora y la excelencia, intentando alcanzar el máximo rendimiento deportivo posible, ha impulsado a la comunidad científica a analizar las diferentes situaciones de juego en un contexto competitivo, mediante el establecimiento de relaciones entre las acciones del juego y la eficacia del mismo, la victoria o derrota de un equipo (Ibáñez y cols. 2003; Karipidis y cols. 2001; Sampaio 2000).

En el deporte del baloncesto, una de las acciones de juego más estudiadas es el lanzamiento, por ser la única acción que permite la obtención del éxito. El lanzamiento a canasta ha sido analizado desde diversos enfoques. Bajo un prisma biomecánico, Rojas y cols. (2002), comprobaron que la presencia de un oponente en el momento del lanzamiento influye en la forma en que éste se desarrolla. Los resultados obtenidos apuntan que, cuando hay un oponente, los jugadores intentan soltar el balón más rápido y desde más altura, justificando la utilización en los entrenamientos de una oposición real en las acciones de lanzamiento. Robbins y cols. (2006), analizaron la influencia que la distancia a la que se lanza tiene con la fuerza que se imprime al lanzamiento. Encontraron relaciones entre la distancia, la fuerza y elángulo de lanzamiento.

Encuadrados en un enfoque de análisis notacional del juego, se encuentran gran cantidad de trabajos en los que el lanzamiento a canasta es relevante. La mayoría de los estudios demuestran la importancia de los lanzamientos en la victoria o derrota de un equipo (Ibáñez y cols 2003; Gómezy cols. 2006; Sampaio y Leite, 2006). Existe un consenso respecto a la importancia mayor de los lanzamientos de 2 puntos sobre el resto de estadísticas de juego en la predicción de pertenencia al grupo de los equipos ganadores o perdedores, junto con el rebote defensivo.

En laúltima década, los trabajos sobre el análisis de los lanzamientos, han comenzado a estudiar otro tipo de variables que inciden en la acción del lanzamiento, y que se producen durante su ejecución, tales

Fecha recepción: 30-01-08 - Fecha envío revisores: 01-02-08 - Fecha de aceptación: 07-04-08 Correspondencia: Javier García Rubio

Avda. de la Universidad s/n

Cáceres (España)

E-mail: jagaru04@alumnos.unex.es como la presión defensiva, el rol del jugador, el nivel de los jugadores, etc... (Ferreira 2001; Ibáñez y cols. 2001a; Ibáñezy cols. 2001b; Ibáñez y cols. 2002; Ortega y cols. 2006). Ortega y cols. (2006) comprueban que la segunda opción más empleada para finalizar las posesiones de balón es el lanzamiento a canasta. La primera opción es el pase, seguidos de las faltas recibidas y los robos de balón. En el caso de la acción previa que realizan los jugadores antes del lanzamiento, Ferreira (2001) encontró que la acción que precede a la mayoría de éstos es el pase o el 1 contra 1. . T11.

También existen diferencias en función del puesto específico que ocupa cada jugador. Algunos trabajos confirman que los pívots empiezan y acaban sus intervenciones muy cercanas a canasta, con un elevado porcentaje de acierto (Papadimitriou y cols. 1999), mientras que los bases realizan penetraciones hacia la canasta (Taxildaris y cols. 2001).

El objeto del presente estudio es profundizar en el estudio del lanzamiento teniendo en cuenta las acciones que se producen durante su ejecución. Para ello se analizará la gestoforma de los lanzamientos a canasta que se producen en la liga E.B.A. (liga española de baloncesto amateur). Este trabajo examina la relación existente entre la gestoforma del tiro a canasta y una serie de variables registradas durante la acción del lanzamiento que describen o definen la situación en la que se ha producido el lanzamiento.

\section{Método}

El diseño de esta investigación según la metodología empleada es cualitativo, descriptivo. Para la recogida de datos la metodología es observacional. Los datos seleccionados para la realización del estudio fueron los lanzamientos que sucedieron durante los partidos de la liga Española de Baloncesto Amateur (E.B.A.). Las variables que se registraron en cada lanzamiento fueron: i) periodo ii) cuarto, ii) eficacia, iv) presión defensiva, v) zona del campo, vi) valor del lanzamiento, vii) rol del jugador que lanza, y viii) la acción previa que realiza. Como variable dependiente se emplea la gestoforma del lanzamiento, siguiendo la propuesta de Ibañez y cols. $(2001 b, 2002)$ para el análisis del lanzamiento. Se estableció un núcleo categorial y los grados de apertura oportunos para la definición de cada una de las variables del estudio (Anguera, 


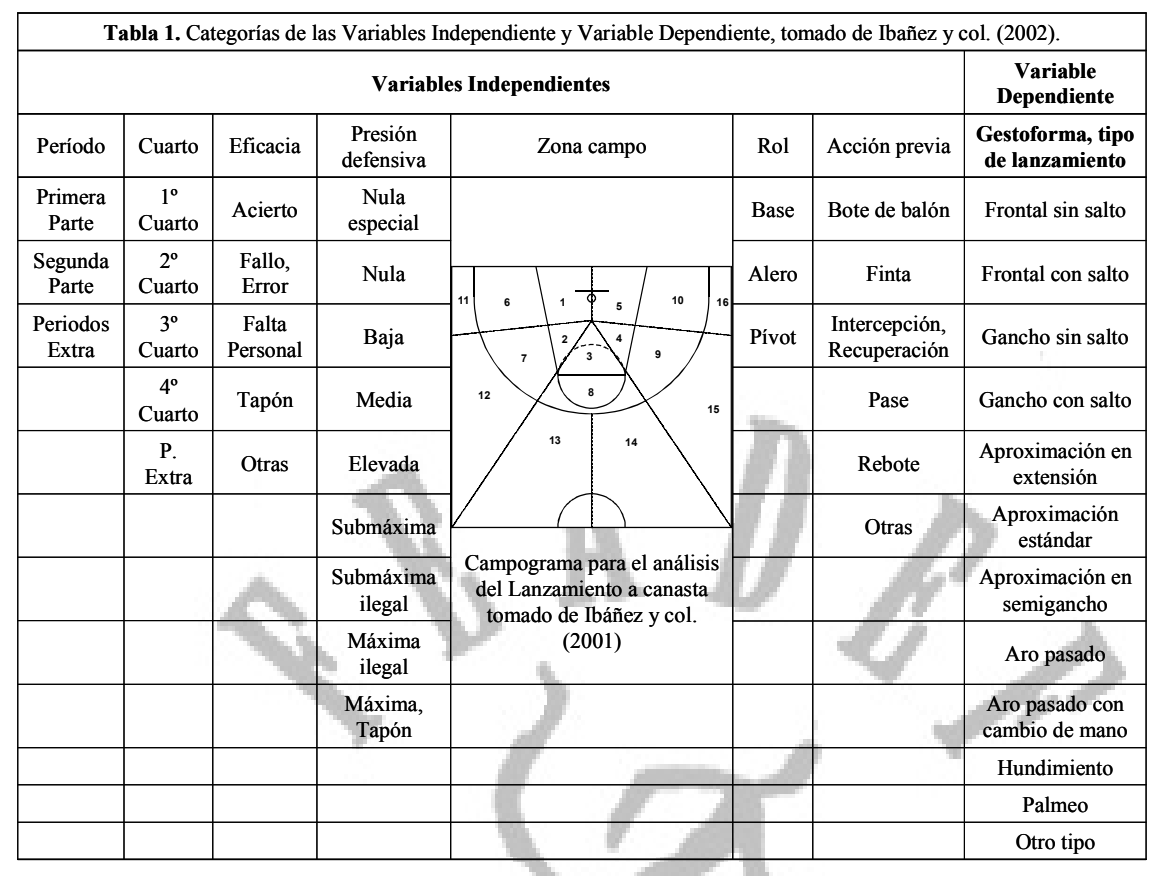

1991). Las categorías/grado de apertura de cada una de las variables, independiente y dependientes, quedan recogidas en la tabla 1.

\subsection{Muestra}

La muestra estuvo constituida por 53 partidos de la liga E.B.A. Se registraron un total de 8869 lanzamientos, siendo la media de 167,40土 21,45 lanzamientos por partido. El $25.6 \%$ de los lanzamientos analizados fueron de 1 punto, el $54 \%$ de 2 puntos y el $20.4 \%$ de 3 puntos.

\subsection{Análisis de datos}

Debido a la naturaleza de los datos (cualitativos) y tras un análisis exploratorio inicial, se emplean modelos matemáticos no paramétricos para el contraste de las hipótesis. Se analizó la asociación entre las variables a partir del Chi cuadrado y Coeficiente de Continencia. La interpretación del grado de asociación se realizó a través de los residuos tipificados corregidos (>1.9) de las tablas de contingencia. Se segmentó el archivo para analizar los lanzamientos de 1 punto y los de campo por separado en base a la investigación previa.

\subsection{Procedimiento de aplicación}

Definidas las variables del estudio, se procedió al entrenamiento de los observadores, mediante una serie de sesiones de formación teórica y práctica. Tras la formación inicial se procedió a calcular el coeficiente de fiabilidad de los datos intra e inter observadores, obteniéndose valores aceptables, superior al $80 \%$ en cada una de las variables (Blázquez, 1990), 90.2\% intra-observador y $89.7 \%$ inter-observadores respectivamente. Los partidos analizados, fueron previamente grabados en video, para un mejor análisis por parte de los observadores. Los datos se introdujeron en el programa estadístico Spss 13.0, para su posterior análisis.

\section{Resultados y discusión}

\subsection{Estadísticos descriptivos}

Lanzamientos de 1 punto. Un total de 2271 lanzamientos se registraron desde la línea de tiros libres, con valor de un punto. Debido a las características especiales de este tipo de lanzamiento, no se tuvo en cuenta para el estudio inferencial. Siempre se lanza desde la misma zona del campo, con la misma presión defensiva, acción previa y valor. La única variable que presenta valores diferentes es el rol del jugador y la eficacia del lanzamiento. La gestoforma predominante es la estándar sin salto, 99.6\%. Ibáñezy cols. (2007a), encontraron una asociación significativa entre los lanzamientos de tiros libre y el rol de jugador que realiza esta acción. A pesar de que los pívots son los jugadores que más lanzamientos realizan de tiros libres, éstos consiguen una eficacia inferior a lo esperado.

Lanzamientos de campo. El restode lanzamientos analizados, 6598, son lanzamientos de campo ( 2 y 3 puntos). El lanzamiento que más se emplea es el estándarcon salto (65.4\%), seguido del lanzamiento estándar sin salto (14.1\%). Le siguen la familia de los lanzamientos tras pasos de aproximación (entradas a canasta), con un $37.8 \%$.

El análisis de la gestoforma del lanzamientos en función de su valor muestra que dentro de los lanzamientos de dos puntos, el 53.2\% son lanzamientos estándar con salto, seguidos de los lanzamientos tras pasos de aproximación en extensión (19.1\%) y tras pasos de aproximación estándar (13.9\%). La gestoforma más empleada en el lanzamiento de tres puntos es el lanzamiento estándar con salto, 97.6\% (Tabla 2).

Ibáñez y cols. (2001a), encontraron que los lanzamientos de 1 punto son realizados fundamentalmente sin salto (98.3\%), mientras que en los lanzamientos de dos puntos las acciones más predominantes son el lanzamiento sin contacto con el suelo $46.7 \%$ (con salto $28.8 \%$ y en suspensión $17.9 \%$ respectivamente), y tras pasos de aproximación en extensión (19.6\%); frente al lanzamiento que se realizan sin contacto del jugador con el suelo, $99 \%$ (con salto $72.2 \%$ y en suspensión $26.8 \%$ ), que se emplea desde más allá de la línea de $6,25 \mathrm{mts}$ ( 3 puntos).

\subsection{Estadística inferencial no paramétrica}

Lanzamientos de campo. No se obtiene relación estadísticamente significativa entre la gestoforma y el periodo de juego $\left(\mathrm{c}^{2}{ }_{(22, \mathrm{~N}=5852)}=\right.$ $19.054, \mathrm{p}>05)$ y cuarto $\left(\mathrm{c}^{2}{ }_{(44, \mathrm{~N}=4186)}=37.605 \mathrm{p}>.05\right)$. No existen diferencias en la forma de lanzar entre la primera y segunda parte, entre los diferentes cuartos, en suma, no hay diferencias a lo largo del partido.

El análisis de la relación entre la gestoforma y la eficacia del lanzamiento, mostró una relación significativa $\left(\mathrm{c}^{2}{ }_{(33, \mathrm{~N}=6598)}=365.287 \mathrm{p}<.01\right)$, con una asociación baja $(C=.229, \mathrm{p}<.01)$. Tras el estudio de los residuos tipificados corregidos se observa que la gestoforma que más posibilidades tiene de conseguir canasta es el lanzamiento tras pasos de aproximación estándar(8.7), seguido del hundimiento(7.7)y del palmeo (4.5). El lanzamiento con menos posibilidad de éxito es el lanzamiento estándar con salto (13.8). El lanzamiento que más posibilidades tiene de recibir falta personal es el lanzamiento tras pasos de aproximación 


\begin{tabular}{|c|c|c|c|c|c|c|c|c|c|c|c|c|c|c|}
\hline \multirow[b]{2}{*}{ VALOR } & & \multicolumn{13}{|c|}{ GESTOFORMA } \\
\hline & & LE SS & LE CS & LG SS & LG CS & $\begin{array}{l}\text { L TPA } \\
\text { EXT }\end{array}$ & $\begin{array}{c}\text { L TPA } \\
\text { EST }\end{array}$ & $\begin{array}{c}\text { L TPA } \\
\text { SGA }\end{array}$ & $\begin{array}{l}\text { L TPA } \\
\text { A PAS }\end{array}$ & $\begin{array}{l}\text { L TPA } \\
\text { A PAS } \\
\text { MNCM }\end{array}$ & MATE & PALM & OTROS & TOTAL \\
\hline \multirow{2}{*}{$\begin{array}{c}\text { Lanzamiento } \\
\text { de } 1 \text { punto }\end{array}$} & $\mathrm{N}$ & 2261 & 10 & 0 & 0 & 0 & 0 & 0 & 0 & 0 & 0 & 0 & 0 & 2271 \\
\hline & $\%$ GEST & $99,3 \%$ &, $2 \%$ &, $0 \%$ &, $0 \%$ &, $0 \%$ &, $0 \%$ &, $0 \%$ &, $0 \%$ &, $0 \%$ &, $0 \%$ &, $0 \%$ &, $0 \%$ & $25,6 \%$ \\
\hline \multirow{2}{*}{$\begin{array}{l}\text { Lanzamiento } \\
\text { de } 2 \text { puntos }\end{array}$} & $\mathrm{N}$ & 11 & 2543 & 19 & 112 & 916 & 665 & 135 & 52 & 43 & 103 & 79 & 108 & 4786 \\
\hline & $\%$ GEST &, $5 \%$ & $58,9 \%$ & $100,0 \%$ & $100,0 \%$ & $98,3 \%$ & $98,4 \%$ & $100,0 \%$ & $100,0 \%$ & $97,7 \%$ & $100,0 \%$ & $100,0 \%$ & $89,3 \%$ & $54,0 \%$ \\
\hline \multirow{2}{*}{$\begin{array}{l}\text { Lanzamiento } \\
\text { de } 3 \text { puntos }\end{array}$} & $\mathrm{N}$ & 5 & 1769 & 0 & 0 & 13 & 11 & 0 & 0 & 0 & 0 & 0 & 14 & 1812 \\
\hline & $\%$ GEST &, $2 \%$ & $41,0 \%$ &, $0 \%$ &, $0 \%$ & $1,4 \%$ & $1,6 \%$ &, $0 \%$ &, $0 \%$ &, $0 \%$ &, $0 \%$ &, $0 \%$ & $11,5 \%$ & $20,4 \%$ \\
\hline \multirow{2}{*}{$\begin{array}{c}\text { Total } \\
\text { lanzamientos }\end{array}$} & $\mathrm{N}$ & 2277 & 4322 & 19 & 112 & 929 & 676 & 135 & 52 & 43 & 103 & 79 & 122 & 8869 \\
\hline & $\%$ total & $25,7 \%$ & $48,7 \%$ & ,2\% & $1,3 \%$ & $10,5 \%$ & $7,6 \%$ & $1,5 \%$ & ,6\% &, $5 \%$ & $1,2 \%$ &, $9 \%$ & $1,4 \%$ & $100,0 \%$ \\
\hline
\end{tabular}

estándar (7.7) y el que menos el lanzamiento estándar con salto (-8.3) (Tabla 3). Los lanzamientos que se realizan de forma dinámica (entradas) se producen cerca de canasta. Estos lanzamientos son los que mayor éxito tienen, a pesar de que la presión defensiva sea más elevada. De hecho, el lanzamiento tras pasos de aproximación estándar es el que más faltas recibe. El lanzamiento más empleado durante el juego, el estándar con salto, es el que más posibilidad tiene de fallarse. Esto implica que hay que mejorar la forma en la que se entrena este tipo de lanzamiento para mejorar la eficacia.

Ibáñez y cols. (2007a) encontraron una relación similar al estudiar los lanzamientos en la liga A.C.B.. Existe una asociación significativa entre la forma de lanzar a canasta y la eficacia del lanzamiento. Esta relación varía en función del nivel de los jugadores analizados.

En el análisis de la relación entre gestoforma y presión defensiva se encontró una relación estadísticamente significativa $\left(\mathrm{c}^{2}{ }_{(77, \mathrm{~N}=6598)}=\right.$ $365.287 \mathrm{p}<.01)$, con un coeficiente de contingencia $C=.229(\mathrm{p}<.01)$. Los residuos tipificados corregidos muestran la relación entre ambas variables. La gestoforma que más posibilidades tiene de efectuarse con una presión defensiva media es el lanzamiento estándar con salto (8.2) (Tabla 4).

La presión defensiva condiciona el tipo de lanzamiento que emplea el jugador. Hay que tener en cuenta la presión defensiva en el entrenamiento del lanzamiento. En categorías de formación, infantiles y cadetes, Ferreira (2001) encontró que en torno al 85\% de los lanzamientos que se realizan tienen presión defensiva. Por su parte, Ortega y cols. (2007), encuentran un porcentaje menor de lanzamientos con presión defensiva, 74\%, al analizar los lanzamientos en jugadores en períodos de formación. En la liga E.B.A., sólo el $4.5 \%$ de los lanzamientos son sin presión defensiva. Del resto, el $13.9 \%$ tienen una presión defensiva baja, el $21.9 \%$ media, el $21.7 \%$ elevada y submáxima el $23.9 \%$.

Posteriormente se estudiaron las relaciones entre la gestoforma y la zona del campo en la que se realiza el lanzamiento, encontrándose una asociación significativa $\left(\mathrm{c}^{2}{ }_{(165, \mathrm{~N}=6598)}=2060.092, \mathrm{p}<.01\right)$, con una asociación media $(C=.488, \mathrm{p}<.01)$. Los residuos tipificados muestran un uso menor de lo esperado en las zonas 1 a 5 para el lanzamiento con salto $(-15.4,-10.0,-8.7,-11.3,-20,2)$, y un uso mayor de lo esperado en el resto, sobre todo en la zona 12 (14.2) y 15 (14.4). Los lanzamientos tras pasos de aproximación en extensión y estándar tienen un porcentaje mayor de lo esperado significativo $(>2)$ en las zonas 1 a 5, y menor de lo esperado en el resto (>-2). En los lanzamientos de tres puntos se encuentra con que las zonas más empleadas son la frontal y central, similar a los resultados encontrados por Ortega y col. (2007), y no en las diagonales (12 y 15). En las zonas diagonales a canasta, paradójicamente, se empela menos el lanzamiento con salto. Los lanzamientos con salto se emplean más cuanto mayor es la distancia respecto a la canasta.

La literatura nos muestra relaciones entre la zona del campo desde la que se realiza el lanzamiento y la eficacia del mismo, tanto en la liga A.C.B. (Ibáñez y cols., 2007a), como en la liga E.B.A. (Ibáñez y cols., 2007b). La forma de lanzar a canasta también está condicionada por la zona y distancia desde la que se lanza. Estos datos hay que tenerlos en cuenta para diseñar sesiones de entrenamiento en las que los jugadores lancen con salto cuando estén en zonas lejanas del aro, y con gran variabilidad de gestoformas y de forma dinámica dentro delárea restringida.

En cuanto a las relaciones entre la gestoforma y el valor del lanzamiento, hay una relación significativa $\left(\mathrm{c}^{2}{ }_{(11, \mathrm{~N}=6598)}=1148.784 \mathrm{p}<.01\right)$, con una asociación media $(C=.385, \mathrm{p}<.01)$. Los residuos tipificados corregidos muestran que en los lanzamientos estándar con salto hay una proporción menor de lo esperada para los lanzamientos de 2 puntos ($33,8)$ y mayor de lo esperado en los lanzamientos de 3 puntos (33.8). Los lanzamientos tras pasos de aproximación tienen mayor posibilidad de efectuarse con valor de dos puntos (en extensión, (15.8); estándar (19.2); semigancho (7.2)) que con valor de tres puntos (en extensión (15.8); estándar(-19.2); semigancho(-7.2)).

El valor del lanzamiento condiciona la forma de lanzar, ya que este valor lleva implícito un aumento de la distancia. Ibáñez y cols. (2001a) indicaban que mientras en los lanzamientos de tiros libres existe una gestoforma casi exclusiva, lanzamiento estándar sin salto, y los lanzamientos frontales a canasta (con o sin salto) son la base de los lanzamientos de tres puntos, la variabilidad era la nota predominante en los lanzamientos de dos puntos. El entrenador debe ofrecer variabilidad a sus jugadores en los ejercicios de lanzamiento, proponiendo gestoformas variadas en los lanzamientos de dos puntos.

El rol del jugädor que lanza presentó una relación significativa con la gestoforma $\left(\mathrm{c}^{2}{ }_{(22, \mathrm{~N}=5394)}=226.979 \mathrm{p}<.01\right)$, con una asociación baja $(C=.201, \mathrm{p}<.01)$. En cuanto a los residuos tipificados corregidos, encontramos que los bases tienen más posibilidades de realizar lanzamientos tras pasos de aproximación en extensión (5.2), y los que menos posibilidades los hundimientos (-3.7) y palmeos (-3.8). Los aleros tienen más posibilidades de realizar los lanzamientos tras pasos de aproximación estándar (3.9) y en extensión (4.9), por el contrario tenemos los lanzamientos en gancho con salto $(-2.8)$ y los lanzamientos estándar con salto (-2.7). Los pívots usan más de lo esperado el gancho con salto (5.7), el hundimiento (5.2) y el palmeo (4.8). Los lanzamientos que menos posibilidades tienen de usar son tras pasos de aproximación en extensión (-9) y estándar (-4.5). Según Ibáñezy cols. (2007), los pívots deben realizar lanzamientos desde zonas cercanas a canasta, con variadas gestoformas y con alta intensidad defensiva. En definitiva, los puestos específicos de juego determinan los lanzamientos que hace cada jugador, debiendo realizar entrenamiento específico para cada rol.

Finalmente, tras comparar la gestoforma y la acción previa, se encontró una relación estadísticamente significativa $\left(\mathrm{c}^{2}{ }_{(55, \mathrm{~N}=4331)}=\right.$ $1307.575 \mathrm{p}<.01)$, con una asociación media $(C=.482, \mathrm{p}<.01)$. El lanzamiento estándar con salto, que es el que más se utiliza, tiene una proporción de uso mayor de lo esperado tras pase (21.5), y menor de lo esperado tras bote (-24.8). El lanzamiento tras pasos de aproximación en extensión tiene más posibilidades de realizarse tras bote (15.5), y menos posibilidades de realizarse tras pase (-11.2). Algo muy parecido ocurre con los lanzamientos tras pasos de aproximación estándar, donde hay una frecuencia mayor de los esperada tras bote (18), y menor tras pase (-13.9) y rebote (-4.2)

Ortega y cols. (2007) identifican un aumento de la eficacia de los lanzamientos de 3 puntos cuando éstos se realizan con pases frontales o desde el lateral derecho de los lanzadores y que sólo un $30.4 \%$ de los 


\begin{tabular}{|c|c|c|c|c|c|c|}
\hline \multirow{2}{*}{\multicolumn{2}{|c|}{ GESTOFORMA }} & \multicolumn{4}{|c|}{ EFICACIA } & \multirow[b]{2}{*}{ Total } \\
\hline & & Acierto & $\begin{array}{l}\text { Fallo } \\
\text { Error }\end{array}$ & $\begin{array}{c}\text { Falta } \\
\text { Personal }\end{array}$ & Tapón & \\
\hline \multirow{3}{*}{$\begin{array}{l}\text { L. Estándar } \\
\text { Sin salto }\end{array}$} & $\mathrm{N}$ & 9 & 4 & 2 & 1 & 16 \\
\hline & $\%$ GEST & $56,3 \%$ & $25,0 \%$ & $12,5 \%$ & $6,3 \%$ & $100,0 \%$ \\
\hline & Res. Corr. & 1,1 & $-1,7$ &, 7 &, 7 & \\
\hline \multirow{3}{*}{$\begin{array}{l}\text { L. Estándar } \\
\text { Con salto }\end{array}$} & $\mathrm{N}$ & 1661 & 2267 & 260 & 124 & 4312 \\
\hline & $\%$ GEST & $38,5 \%$ & $52,6 \%$ & $6,0 \%$ & $2,9 \%$ & $100,0 \%$ \\
\hline & Res. Corr. & $-8,6$ & 13,8 & $-8,3$ & $-2,1$ & \\
\hline \multirow{3}{*}{$\begin{array}{c}\text { L. Gancho } \\
\text { Sin salto }\end{array}$} & $\mathrm{N}$ & 15 & 2 & 0 & 2 & 19 \\
\hline & $\%$ GEST & $78,9 \%$ & $10,5 \%$ &, $0 \%$ & $10,5 \%$ & $100,0 \%$ \\
\hline & Res. Corr. & 3,2 & $-3,1$ & $-1,3$ & 1,8 & \\
\hline \multirow{3}{*}{$\begin{array}{l}\text { L. Gancho } \\
\text { Con salto }\end{array}$} & $\mathrm{N}$ & 55 & 44 & 6 & 7 & 112 \\
\hline & $\%$ GEST & $49,1 \%$ & $39,3 \%$ & $5,4 \%$ & $6,3 \%$ & $100,0 \%$ \\
\hline & Res. Corr. & 1,5 & $-1,5$ & $-1,1$ & 1,8 & \\
\hline \multirow{3}{*}{$\begin{array}{l}\text { L. Tras pasos } \\
\text { Aproximación } \\
\text { Extensión }\end{array}$} & $\mathrm{N}$ & 515 & 276 & 105 & 33 & 929 \\
\hline & $\%$ GEST & $55,4 \%$ & $29,7 \%$ & $11,3 \%$ & $3,6 \%$ & $100,0 \%$ \\
\hline & Res. Corr. & 8,7 & $-11,0$ & 3,9 & 6 & \\
\hline \multirow{3}{*}{$\begin{array}{c}\text { L. Tras pasos } \\
\text { Aproximación } \\
\text { Estandard }\end{array}$} & $\mathrm{N}$ & 259 & 281 & 106 & 30 & 676 \\
\hline & $\%$ GEST & $38,3 \%$ & $41,6 \%$ & $15,7 \%$ & $4,4 \%$ & $100,0 \%$ \\
\hline & Res. Corr. & $-2,2$ & $-2,7$ & 7,7 & 1,9 & \\
\hline \multirow{3}{*}{$\begin{array}{c}\text { L. Tras pasos } \\
\text { Aproximación } \\
\text { Semigancho }\end{array}$} & $\mathrm{N}$ & 51 & 63 & 17 & 4 & 135 \\
\hline & $\%$ GEST & $37,8 \%$ & $46,7 \%$ & $12,6 \%$ & $3,0 \%$ & $100,0 \%$ \\
\hline & Res. Corr. & $-1,1$ &, 1 & 2,0 &,- 2 & \\
\hline \multirow{3}{*}{$\begin{array}{c}\text { L. Tras pasos } \\
\text { Aproximación } \\
\text { Aro pasado }\end{array}$} & $\mathrm{N}$ & 22 & 24 & 4 & 2 & 52 \\
\hline & $\%$ GEST & $42,3 \%$ & $46,2 \%$ & $7,7 \%$ & $3,8 \%$ & $100,0 \%$ \\
\hline & Res. Corr. & 0 & 0 &,- 1 &, 3 & \\
\hline \multirow{3}{*}{$\begin{array}{l}\text { L. Tras pasos } \\
\text { Aproximación } \\
\text { A-p mano cam }\end{array}$} & $\mathrm{N}$ & 22 & 17 & 3 & 1 & 43 \\
\hline & $\%$ GEST & $51,2 \%$ & $39,5 \%$ & $7,0 \%$ & $2,3 \%$ & $100,0 \%$ \\
\hline & Res. Corr. & 1,2 &,- 9 &,- 3 &,- 3 & \\
\hline \multirow{3}{*}{ Hundimiento } & $\mathrm{N}$ & 82 & 13 & 5 & 3 & 103 \\
\hline & $\%$ GEST & $79,6 \%$ & $12,6 \%$ & $4,9 \%$ & $2,9 \%$ & $100,0 \%$ \\
\hline & Res. Corr. & 7,7 & $-6,9$ & $-1,2$ &,- 2 & \\
\hline \multirow{3}{*}{ Palmeo } & $\mathrm{N}$ & 53 & 22 & 3 & 1 & 79 \\
\hline & $\%$ GEST & $67,1 \%$ & $27,8 \%$ & $3,8 \%$ & $1,3 \%$ & $100,0 \%$ \\
\hline & Res. Corr. & 4,5 & $-3,3$ & $-1,4$ & $-1,0$ & \\
\hline \multirow{3}{*}{ Otros } & $\mathrm{N}$ & 49 & 48 & 21 & 4 & 122 \\
\hline & $\%$ GEST & $40,2 \%$ & $39,3 \%$ & $17,2 \%$ & $3,3 \%$ & $100,0 \%$ \\
\hline & Res. Corr. &,- 5 & $-1,6$ & 3,7 & 0 & \\
\hline \multirow{2}{*}{ TOTAL } & $\mathrm{N}$ & 2793 & 3061 & 532 & 212 & 6598 \\
\hline & $\%$ GEST & $42,3 \%$ & $46,4 \%$ & $8,1 \%$ & $3,2 \%$ & $100,0 \%$ \\
\hline
\end{tabular}

hay que enseñarlo y entrenarlo con la gestoforma más eficiente y que permite una mayor eficacia, el lanzamiento estándar sin salto. En segundo lugar, tenemos que tener en cuenta el volumen de lanzamientos y la proporción de los mismos que se entrena en las sesiones. Se deberán establecer protocolos para el entrenamiento de este tipo de lanzamiento de forma continua y progresiva durante el entrenamiento (Ibáñez y cols. 2007b).

El lanzamiento más empleado en la acción de juego es el lanzamiento estándar con salto, por tanto, este tipo de lanzamiento tendrá un tratamiento especial durante las sesiones de entrenamiento. Este tipo de lanzamiento tiene más relevancia cuando aumenta la distancia respecto a canasta. Además, cuando se diseñe un ejercicio para entrenar un tipo de lanzamiento, hay que tener en cuenta que debe realizarse con presión defensiva. En el entrenamiento del lanzamiento, el entrenador debe modular la presión defensiva, ajustándola a la distancia del lanzador y a la gestoforma empleada. Los resultados de esta investigación indican que para adaptar el entrenamiento del lanzamiento a la competición, cuando se entrene el lanzamiento estándar sin salto hay que hacerlo con presión defensiva media, es decir un compañero delante, $y$ teniendo en cuenta la zona del campo donde más posibilidades tiene de realizarse. Los lanzamientos a canasta tras pasos de aproximación (toda la familia de este tipo de lanzamientos) hay que entrenarlos desde dentro de la zona de tres segundos para ser eficaz (en cualquiera de sus cinco zonas), y con una alta presión defensiva, pues hay muchas posibilidades de en el juego real se realice con las oposición de defensores (Ibáñezy cols. 2007 a; Ibáñez y cols. 2007b). Por este motivo, las tradicionales ruedas de entradas a canasta no tendrán una aplicación en la competición, pues no tienen transferencia real en el juego.

Del análisis de las zonas del campo desde las que se ejecuta el lanzamiento, se pone de manifiesto la necesidad de incrementar el volumen de ejecuciones que se realicen desde las zonas más usadas por los jugadores, y con la gestoforma más habitual. Por ejemplo, incrementar los lanzamientos de tres puntos desde cada una de las diagonales al aro, zonas 12 y 15 . Por otro lado, dentro del área restringida los lanzamientos estándar sin salto no son muy empleados, siendo más recomendables los lanzamientos tras pasos de aproxi-

lanzamientos están precedidos de alguna acción técnico-táctica. Estos resultados ratifican la importancia de tener en cuenta la acción previa que realizan los jugadores antes de lanzar. En el entrenamiento del lanzamiento a canasta debe existir variabilidad en la acción que lo precede. Tradicionalmente existe una cierta monotonía en esta variable, con un gran predominio del pase, que debe alternarse con otras acciones.

\section{Conclusiones}

Este trabajo analiza el lanzamiento que realizan los jugadores durante la competición real, la Liga EBA, con el objetivo de conocer cómo se producen las acciones de juego en un contexto real. Este trabajo se adapta a las necesidades y tendencias de investigación en el estudio de la acción de juego en baloncesto (Ibáñez y cols. 2001b). A partir de los resultados sobre las características de la gestoforma del lanzamiento, se deberán planificar los entrenamientos, intentando que los ejercicios se adecuen a las especifidades de la competición y nivel de los jugadores (Ferreira, 2001; Ortega y cols. 2007).

El tiro libre es una de las acciones más importantes en el desarrollo del juego, aproximadamente un $25 \%$ del total de lanzamientos. Por ello, mación.

Es importante saber qué tipo de lanzamiento realiza cada tipo de jugador, pues así se podrá individualizar el trabajo (Papadimitrio y cols. 1999). Los resultados de este estudio nos indican que debe existir un predominio en el entrenamiento de los bases de los lanzamientos tras pasos de aproximación, teniendo menos relevancia los palmeos y hundimientos o mates. Los aleros presentan un perfil de uso de los lanzamientos parecido, con los lanzamientos tras pasos de aproximación estándar y en extensión, debido a la gran cantidad de entradas a canasta y contraataques que realizan. Los pívots, el lanzamiento que más utilizan es el gancho con salto, debido a que los lanzamientos son todos con mucha presión defensiva al estar cerca de canasta, también hay un uso elevado de los palmeos.

En cuanto a la acción previa, los entrenadores deben tener en cuenta que existen acciones diferentes que preceden a los lanzamientos. La variabilidad en este tipo de acciones será fundamental para conseguir un entrenamiento más adaptado a las necesidades y realidades de la competición (Ibáñez y cols. 2007b; Ortega y cols. 2007). Para el diseño de los ejercicios que en la situación real de juego de los lanzamientos estándar en salto se emplearán con más frecuencia acciones previas de pase y 


\begin{tabular}{|c|c|c|c|c|c|c|c|c|c|c|}
\hline \multirow{2}{*}{\multicolumn{2}{|c|}{ GESTOFORMA }} & \multicolumn{9}{|c|}{ PRESIÓN DEFENSIVA } \\
\hline & & Nula & Baja & Media & Elevada & $\begin{array}{c}\text { Sub } \\
\text { máxima }\end{array}$ & $\begin{array}{c}\text { Sub } \\
\text { máxima } \\
\text { Ilegal }\end{array}$ & $\begin{array}{c}\text { Máxima } \\
\text { ilegal }\end{array}$ & $\begin{array}{l}\text { Máxima } \\
\text { Tapon }\end{array}$ & Total \\
\hline \multirow{3}{*}{$\begin{array}{l}\text { L. Estándar } \\
\text { Sin salto }\end{array}$} & $\mathrm{N}$ & 3 & 2 & 4 & 2 & 2 & 0 & 2 & 1 & 16 \\
\hline & $\%$ GEST & $18,8 \%$ & $12,5 \%$ & $25,0 \%$ & $12,5 \%$ & $12,5 \%$ &, $0 \%$ & $12,5 \%$ & $6,3 \%$ & $100,0 \%$ \\
\hline & Res. Corr. & 2,7 &,- 2 & ,3 &,- 9 & $-1,1$ &,- 7 &, 7 & ,7 & \\
\hline \multirow{3}{*}{$\begin{array}{l}\text { L. Estándar } \\
\text { Con salto }\end{array}$} & $\mathrm{N}$ & 212 & 672 & 1074 & 865 & 1021 & 88 & 256 & 124 & 4312 \\
\hline & $\%$ GEST & $4,9 \%$ & $15,6 \%$ & $24,9 \%$ & $20,1 \%$ & $23,7 \%$ & $2,0 \%$ & $5,9 \%$ & $2,9 \%$ & $100,0 \%$ \\
\hline & Res. Corr. & 2,1 & 5,6 & 8,2 & $-4,5$ &,- 6 & $-5,8$ & $-8,5$ & $-2,1$ & \\
\hline \multirow{3}{*}{$\begin{array}{l}\text { L. Gancho } \\
\text { Sin salto }\end{array}$} & $\mathrm{N}$ & 0 & 2 & 1 & 8 & 4 & 2 & 0 & 2 & 19 \\
\hline & $\%$ GEST &, $0 \%$ & $10,5 \%$ & $5,3 \%$ & $42,1 \%$ & $21,1 \%$ & $10,5 \%$ &, $0 \%$ & $10,5 \%$ & $100,0 \%$ \\
\hline & Res. Corr. & $-1,0$ &,- 4 & $-1,8$ & 2,2 &,- 3 & 2,0 & $-1,3$ & 1,8 & \\
\hline \multirow{3}{*}{$\begin{array}{l}\text { L. Gancho } \\
\text { Con salto }\end{array}$} & $\mathrm{N}$ & 0 & 3 & 12 & 38 & 41 & 5 & 6 & 7 & 112 \\
\hline & $\%$ GEST &, $0 \%$ & $2,7 \%$ & $10,7 \%$ & $33,9 \%$ & $36,6 \%$ & $4,5 \%$ & $5,4 \%$ & $6,3 \%$ & $100,0 \%$ \\
\hline & Res. Corr. & $-2,3$ & $-3,5$ & $-2,9$ & 3,2 & 3,2 & 1,0 & $-1,0$ & 1,8 & \\
\hline \multirow{3}{*}{$\begin{array}{c}\text { L. Tras pasos } \\
\text { Aproximación } \\
\text { Extensión }\end{array}$} & $\mathrm{N}$ & 47 & 137 & 159 & 216 & 183 & 46 & 108 & 33 & 929 \\
\hline & $\%$ GEST & $5,1 \%$ & $14,7 \%$ & $17,1 \%$ & $23,3 \%$ & $19,7 \%$ & $5,0 \%$ & $11,6 \%$ & $3,6 \%$ & $100,0 \%$ \\
\hline & Res. Corr. & 8 & ,9 & $-3,8$ & 1,2 & $-3,2$ & 4,0 & 4,4 & 6 & \\
\hline \multirow{3}{*}{$\begin{array}{l}\text { L. Tras pasos } \\
\text { Aproximación } \\
\text { Estándar }\end{array}$} & $\mathrm{N}$ & 14 & 53 & 105 & 171 & 162 & 38 & 103 & 30 & 676 \\
\hline & $\%$ GEST & $2,1 \%$ & $7,8 \%$ & $15,5 \%$ & $25,3 \%$ & $24,0 \%$ & $5,6 \%$ & $15,2 \%$ & $4,4 \%$ & $100,0 \%$ \\
\hline & Res. Corr. & $-3,2$ & $-4,8$ & $-4,2$ & 2,4 &, 0 & 4,4 & 7,3 & 1,9 & \\
\hline \multirow{3}{*}{$\begin{array}{l}\text { L. Tras pasos } \\
\text { Aproximación } \\
\text { Semigancho }\end{array}$} & $\mathrm{N}$ & 0 & 7 & 26 & 39 & 38 & 4 & 17 & 4 & 135 \\
\hline & $\%$ GEST &, $0 \%$ & $5,2 \%$ & $19,3 \%$ & $28,9 \%$ & $28,1 \%$ & $3,0 \%$ & $12,6 \%$ & $3,0 \%$ & $100,0 \%$ \\
\hline & Res. Corr. & $-2,6$ & $-2,9$ &,- 7 & 2,0 & 1,2 &, 0 & 2,0 &,- 2 & \\
\hline \multirow{3}{*}{$\begin{array}{c}\text { L. Tras pasos } \\
\text { Aproximación } \\
\text { Aro pasado }\end{array}$} & $\mathrm{N}$ & 0 & 3 & 10 & 10 & 22 & 1 & 4 & 2 & 52 \\
\hline & $\%$ GEST &, $0 \%$ & $5,8 \%$ & $19,2 \%$ & $19,2 \%$ & $42,3 \%$ & $1,9 \%$ & $7,7 \%$ & $3,8 \%$ & $100,0 \%$ \\
\hline & Res. Corr. & $-1,6$ & $-1,7$ &,- 5 &,- 4 & 3,1 &,- 4 &,- 1 &, 3 & \\
\hline \multirow{3}{*}{$\begin{array}{l}\text { L. Tras pasos } \\
\text { Aproximación } \\
\text { A-p mano cam }\end{array}$} & $\mathrm{N}$ & 0 & 2 & 3 & 11 & 21 & 2 & 3 & 1 & 43 \\
\hline & $\%$ GEST &, $0 \%$ & $4,7 \%$ & $7,0 \%$ & $25,6 \%$ & $48,8 \%$ & $4,7 \%$ & $7,0 \%$ & $2,3 \%$ & $100,0 \%$ \\
\hline & Res. Corr. & $-1,4$ & $-1,8$ & $-2,4$ & 6 & 3,8 &, 7 &,- 2 &,- 3 & \\
\hline \multirow{3}{*}{ Hundimiento } & $\mathrm{N}$ & 15 & 23 & 23 & 15 & 17 & 2 & 5 & 3 & 103 \\
\hline & $\%$ GEST & $14,6 \%$ & $22,3 \%$ & $22,3 \%$ & $14,6 \%$ & $16,5 \%$ & $1,9 \%$ & $4,9 \%$ & $2,9 \%$ & $100,0 \%$ \\
\hline & Res. Corr. & 4,9 & 2,5 &, 1 & $-1,8$ & $-1,8$ &,- 6 & $-1,2$ &,- 2 & \\
\hline \multirow{3}{*}{ Palmeo } & $\mathrm{N}$ & 3 & 4 & 14 & 33 & 20 & 1 & 3 & 1 & 79 \\
\hline & $\%$ GEST & $3,8 \%$ & $5,1 \%$ & $17,7 \%$ & $41,8 \%$ & $25,3 \%$ & $1,3 \%$ & $3,8 \%$ & $1,3 \%$ & $100,0 \%$ \\
\hline & Res. Corr. &,- 3 & $-2,3$ &,- 9 & 4,3 &, 3 &,- 9 & $-1,4$ & $-1,0$ & \\
\hline \multirow{3}{*}{ Otros } & $\mathrm{N}$ & 5 & 6 & 11 & 26 & 46 & 3 & 21 & 4 & 122 \\
\hline & $\%$ GEST & $4,1 \%$ & $4,9 \%$ & $9,0 \%$ & $21,3 \%$ & $37,7 \%$ & $2,5 \%$ & $17,2 \%$ & $3,3 \%$ & $100,0 \%$ \\
\hline & Res. Corr. &,- 2 & $-2,9$ & $-3,5$ &,- 1 & 3,6 &,- 3 & 3,8 &, 0 & \\
\hline \multirow{2}{*}{ TOTAL } & $\mathrm{N}$ & 299 & 914 & 1442 & 1434 & 1577 & 192 & 528 & 212 & 6598 \\
\hline & $\%$ GEST & $4,5 \%$ & $13,9 \%$ & $21,9 \%$ & $21,7 \%$ & $23,9 \%$ & $2,9 \%$ & $8,0 \%$ & $3,2 \%$ & $100,0 \%$ \\
\hline
\end{tabular}

Ibáñez, S. J., Lozano, A. y Martínez, B. (2001b). Líneas de investigación en el análisis de las acciones de juego en baloncesto. En S.J. Ibáñez y M.M. Macías Aportaciones al proceso de enseñanza y entrenamiento del baloncesto. Cáceres: Copegraf s/l.

Ibáñez, S. J., Martínez, B., Feu, S. y Lozano, A. (2002). Estudio de los gestos técnico-tácticos en situación real de juego en baloncesto. Trabajo presentado en el II Congreso de Ciencias del Deporte. Madrid

Ibáñez, S. J., Sampaio, J., Sáenz-López, P., Giménez, J. y Janeira, M. A. (2003). Game statistics discriminating the final outcome of Junior World Basketball Championship matches (Portugal 1999). Journal of Human Movement Studies, 45(1), 1-19.

Ibáñez, S. J., García, J., Cañadas, M., Parejo, I. y Feu, S. (2007a). Multifactorial study of shot efficacy in the Spanish professional basketball league. Trabajo presentado en el IV Congreso Ibérico de Baloncesto. Cáceres

Ibáñez, S.J., García, J., Cañadas, M., Moreno, M.I., Lorenzo, A. y Gómez, M.A. (2007b). Estudio de la eficacia del lanzamiento a canasta en la liga e.b.a.. Revista portuguesa de ciencias do desporto, 7 (sup.1), 78.

Karipidis, A., Fotinakis, P., Taxildaris, K. y Fatouros, J. (2001). Factors characterizing a successful performance in basketball. Journal of Human Movement Studies, 41(5), 385-397.

Mexas, K., Tsitskaris, G., Kyriakou, D. y Garefis, A. (2005). Comparison of effectiveness of organized offences between two different championships in high level basketball. International Journal of Performance Analysis in Sport, 5, 72-82.

Ortega, E., Cárdenas, D., Sainz de Baranda P. y Palao J. M. (2006). Analysis $f$ the final action used in basketball during formative years according to

por el contrario en los lanzamientos tras pasos de aproximación se realizan con más frecuencia tras bote.

Se manifiesta la utilidad de estos estudios, tanto por su aplicabilidad en los entrenamientos como por su posterior eficacia para el juego. Lo que lleva a realizar análisis más profundos de éstas y demás acciones del juego.

\section{Referencias}

Anguera, M. T. (1991). Metodología observacional en la investigación psicológica. Volumen I, Fundamentación. Barcelona: Ed. Promociones y Publicaciones universitarias.

Blázquez, D. (1990). Evaluar en educación fisica. Barcelona: Inde.

Ferreira, A. P. (2001). Observaçao multidimensional de lançamento. Uma análise comparativa entre os escalóes de iniciados e cadetes. En S.J. Ibáñez y M.M. Macías Aportaciones al proceso de enseñanza y entrenamiento del baloncesto. Cáceres: Copegraf s/l.

Gómez, M. A., Lorenzo, A., Sampaio, J. y Ibáñez, S. J. (2006). Differences in game-related statistics between winning and losing teams in women's basketball. Journal of Human Movement Studies, 51, 357-369.

Ibáñez, S. J., Lozano, A. y Martinez, B. (2001a). Análisis del tiro a canasta en función del tipo y valor de los lanzamientos, género y nivel de los jugadores. En F. Tavares et al. Tendências actuais investigação em basquetebol (159-172). Porto: Ediciones FCDEF. player's position. Journal of Human Movements Studies, 50 (421437).

Ortega, E., Fernández, R., Gómez, M.A., Lorenzo, A., Ibáñez, S.J. y Sampaio, J. (2007). Análisis de las variables que anteceden al lanzamiento de 3 puntos en baloncesto de alto rendimiento y la repercusión en su eficacia. Revista portuguesa de ciencias do desporto, 7 (sup.1), 35.

Papadimitriou, K., Taxildaris, K., Derri, V. y Mantis, K. (1999). Profile of different level basketball centers. Journal of Human Movement Studies, 37(2), 87-105.

Robbins, M., Wheat, J., Irwin G. y Bartlett R. (2006). The effect of shotting distance on movement variability in basketball. Journal of Human Movements Studies, 50, 217-238.

Rojas, F. J., Cepero, M., Oña, A. y Gutiérrez, M. (2000). Kinematic adjustments in the basketball jump shot against an opponent. Ergonomics, 43, 1651-1660.

Sampaio, A. (2000). O poder Discriminatorio das Estatísticas Do Jogo De Basquetebol Em Diferentes Contextos. Novos Caminos Metodológicos De Análise. Unpublished Dissertaçao De Doctouramento., UTAD, Vila Real.

Sampaio, J. y Leite, N. (2006). ¿Por qué ganaron o perdieron los partidos de baloncesto los equipos que participaron en el Eurobasket 2005? Kronos, 9, 67-73.

Taxildaris, K., Papadimitriou, K., Alexopoulos, P., Fatouros, I. G., Kambas, A., Karipidis, A., Aggelousis, N. y Barbas, I.. (2001). Factors characterizing the offensive game of the playmaker position in basketball. Journal of Human Movement Studies, 40(6), 405-421. 